\title{
Correction to: The IgG galactosylation ratio is higher in spondyloarthritis patients and associated with the MRI score
}

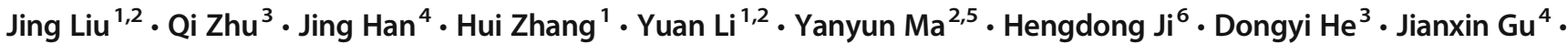 \\ Xiaodong Zhou ${ }^{7} \cdot$ John D. Reveille ${ }^{7} \cdot$ Li Jin $^{1,2} \cdot$ Hejian Zou ${ }^{8,9} \cdot$ Shifang Ren ${ }^{4}$. Jiucun Wang ${ }^{1,2,9}$
}

Published online: 5 August 2020

(C) International League of Associations for Rheumatology (ILAR) 2020

\section{Correction to: Clinical Rheumatology (2020) 39:2317-2323 https://doi.org/10.1007/s10067-020-04998-5}

In the original version of the above article the References 19 and 20 were incorrect which cannot describe the development of the SPARCC score.

The original text reads, "Inflammation index of sacroiliac and spine, and structure score of sacroiliac joint by MIR according to criteria of Spondyloarthritis Research Consortium of Canada (SPARCC) [18-20]." Therefore, the authors would like to substitute References 19 and 20, and added reference 3. The text will now read as "Inflammation index of sacroiliac and spine, and structure score of sacroiliac joint by MIR according to criteria of Spondyloarthritis Research Consortium of Canada (SPARCC) [18-21]." The correct references are as follows:

The authors appreciated Prof.Maksymowych for pointing out the incorrect references.

The online version of the original article can be found at https://doi.org/ 10.1007/s10067-020-04998-5

Hejian Zou

hjzou@fudan.eud.cn

Shifang Ren

shifangren@fudan.edu.cn

Jiucun Wang

jcwang@fudan.edu.cn

1 State Key Laboratory of Genetic Engineering, Collaborative Innovation Center for Genetics and Development, School of Life Sciences, Fudan University, Shanghai, China

2 Human Phenome Institute, Fudan University, Shanghai, China

3 Institute of Arthritis Research, Shanghai Academy of Chinese Medical Sciences, Guanghua Integrative Medicine Hospital, Shanghai, China

\section{References}

19. Maksymowych WP, Inman RD, Salonen D et al (2005) Spondyloarthritis research Consortium of Canada magnetic resonance imaging index for assessment of sacroiliac joint inflammation in ankylosing spondylitis. Arthritis Rheum 53(5):703-9 20. Maksymowych WP, Inman RD, Salonen D et al (2005) Spondyloarthritis Research Consortium of Canada magnetic resonance imaging index for assessment of spinal inflammation in ankylosing spondylitis. Arthritis Rheum 53(4):502-9 21. Maksymowych WP, Wichuk S, Chiowchanwisawakit $P$, Lambert RG, Pedersen SJ (2015) Development and preliminary validation of the spondyloarthritis research consortium of Canada magnetic resonance imaging sacroiliac joint structural score. J Rheumatol 42(1):79-86

Publisher's note Springer Nature remains neutral with regard to jurisdictional claims in published maps and institutional affiliations.

4 Department of Biochemistry andMolecular Biology, Key Laboratory of Glycoconjugate Research Ministry of Public Health, School of Basic Medical Sciences, Fudan University, Shanghai, China

5 Ministry of Education Key Laboratory of Contemporary Anthropology, Department of Anthropology and Human Genetics, School of Life Sciences, Fudan University, Shanghai, China

6 Division of Rheumatology, Taizhou People's Hospital, Taizhou, Jiangsu Province, China

7 Division of Rheumatology and Clinical Immunogenetics, The University of Texas-McGovern Medical School, Houston, TX, USA

8 Division of Rheumatology, Huashan Hospital, Fudan University, Shanghai, China

9 Institute of Rheumatology, Immunology and Allergy, Fudan University, Shanghai, China 